\title{
Design of Two-Channel Linear-Phase FIR PR Filter Banks with Even Length Filters Using Convolution Matrices
}

\author{
A. Muthuvel and Anamitra Makur, Senior Member, IEEE
}

\begin{abstract}
In this paper, the design problem of two-channel linear-phase finite-impulse response perfect reconstruction filter banks in which both the filters are of even length (which are known as type $A$ filter banks) is addressed. The condition on the determinant of the polyphase matrix is translated in terms of well-known convolution matrices. The perfect reconstruction condition is obtained as a system of linear equations. Using the derived condition, an algorithm for the design of type $A$ filter bank by searching the entire class in a sequential approach for both equal and unequal length cases is presented. In this approach, the first analysis filter is chosen such that there exists a filter forming a type $A$ system. The second analysis filter is then chosen using the remaining degrees of freedom. These two steps are iterated, leading to joint optimization of both the filters.
\end{abstract}

Index Terms-Convolution matrix, linear phase filter bank, perfect reconstruction, sequential characterization, two-channel filter bank.

\section{INTRODUCTION}

$\mathbf{T}$ WO-CHANNEL finite-impulse response (FIR) perfect reconstruction (PR) filter banks have been extensively studied in the literature because of their important role in signal decomposition. In this class of filter banks, the subclass of linear-phase filter banks has received considerable attention in the recent past because of its suitability to some applications. In an outstanding work [1], the authors enumerate all possible combinations of the parities of the filter lengths and symmetry of the filters that lead to nontrivial filters. Accordingly, they classify nontrivial linear-phase PR filter banks into two types: type $A$ (one filter is symmetric and another antisymmetric, and both are of even length) and type $B$ (both filters are of odd length and symmetric). Lattice characterizations, derived from the well-known linear prediction lattice, have been given for both types. The characterizations are incomplete (although the authors observed that the loss of generality because of the incompleteness is negligible) and do not lead to a parameterization that allow control over the lengths of the individual filters. The characterization derived using different approaches in [2] and [3] coincides with the characterization given in [1] for type $A$ filter banks.

Manuscript received August 1999; revised August 2000. This paper was recommended by Associate Editor J. LeBlanc.

A. Muthuvel was with the Department of Electrical Communication Engineering, Indian Institute of Science, Bangalore, India. He is now with the Swiss Federal Institute of Technology, Lausanne, Switzerland.

A. Makur is with the Department of Electrical Communication Engineering, Indian Institute of Science, Bangalore, India.

Publisher Item Identifier S 1057-7130(00)11025-0.
In [4], the authors propose a design approach in which the parameterization is obtained by symbolic computation for each possible combination of filter lengths.

Given $K$ filters, the problem of finding $M-K$ complementary filters such that they form an $M$-channel FIR perfect reconstruction filter bank is addressed in [5]. The authors first derive a condition that the first $K$ filters should satisfy for the existence of complementary filters, and then characterize the complementary filters using Smith form decomposition. This condition for type $A$ filter banks is that the polyphase component of the given filter should not have roots on the unit circle. In that case, the authors verify that the complementary filter of the same length is unique. However, their characterization with joint optimization of both the filters becomes complex because of the Smith form decomposition.

In [6], for a given analysis low-pass filter, the synthesis low-pass filter of a type $A$ filter bank longer than the analysis lowpass filter is obtained by a null space projection method using the remaining degrees of freedom. Similar method is given for type $B$ filter banks in [7]. Since only low-pass filters (analysis and synthesis) are allowed in the optimization, the method is not suitable for cost functions involving both the analysis filters (like subband coding).

In [8], the authors give complete characterizations in terms of some canonical sets of parameters for both type $A$ and type $B$ filter banks. Since for some given filter lengths, the number and the cardinality of these sets is not fixed, a parameterization similar to [1] is not feasible. Instead, the authors propose a sequential design, where the first filter is chosen using the Remez algorithm. The canonical sets of parameters are then determined using the so-called identification algorithm. The second filter is then chosen using the remaining degrees of freedom. However, the approach is not adoptable for joint optimization of both the filters because of the complexity of the identification algorithm.

In [9], a characterization has been given for multiplierless two-channel linear-phase FIR perfect reconstruction filter banks. Design methods based on constrained optimization for type $A$ and type $B$ filter banks with equal or unequal length filters have been provided in [10]. However, the matrices involved in the algorithms have to be derived for each combination of filter lengths. A design method based on constrained optimization for type $A$ filter banks with equal length filters is given in [11].

In this paper, we develop a sequential characterization which allows design via optimization by searching the entire class of type $A$ filter banks. Further, the method allows control over the 
lengths of the individual filters. In Section II, a characterization in terms of the convolution matrix is derived. In Section III, a design algorithm using the characterization is presented. Design examples are presented in Section IV. Section V concludes the paper.

\section{A. Notations and Conventions}

Bold-faced and calligraphic upper-case letters represent matrices and bold-faced lower-case letters represent vectors. I and $\mathbf{J}$ with single subscript denote identity and counter identity matrices with the subscript denoting the dimension. $\mathbf{0}$ with double subscript is used to denote zero matrices (either rectangular or square) with the subscripts denoting the dimensions. A length $k+1$ polynomial $A_{k}(z)=\sum_{i=0}^{k} z^{-i} a_{i}$ is alternatively represented as $A_{k}(z)=\mathbf{a}_{k}^{T} \mathbf{e}_{k}(z)$ where $\mathbf{a}_{k}=\left[\begin{array}{llll}a_{0} & a_{1} & \ldots & a_{k}\end{array}\right]^{T}$ and $\mathbf{e}_{k}(z)=\left[\begin{array}{llll}1 & z^{-1} & \ldots & z^{-k}\end{array}\right]^{T}$. $\tilde{\mathbf{a}}_{k}$ represents the reversed version of the vector $\mathbf{a}_{k}$, i.e., $\tilde{\mathbf{a}}_{k}=\left[\begin{array}{llll}a_{k} & a_{k-1} & \ldots & a_{0}\end{array}\right]^{T}$. Then $\tilde{A}_{k}(z)=z^{-k} A_{k}\left(z^{-1}\right)=\tilde{\mathbf{a}}_{k}^{T} \mathbf{e}_{k}(z) \cdot H_{\left[\mathbf{a}_{k}: s\right]}(z)$ denotes the length $2 k+2$ symmetric linear-phase filter with $\mathbf{a}_{k}^{T} \mathbf{e}_{k}(z)$ and $\tilde{\mathbf{a}}_{k}^{T} \mathbf{e}_{k}(z)$ as the first and second polyphase components, i.e., $H_{\left[\mathbf{a}_{k}: s\right]}(z)=\mathbf{a}_{k}^{T} \mathbf{e}_{k}\left(z^{2}\right)+z^{-1} \tilde{\mathbf{a}}_{k}^{T} \mathbf{e}_{k}\left(z^{2}\right) . H_{\left[\mathbf{a}_{k}: a\right]}(z)$ denotes the length $2 k+2$ antisymmetric linear-phase filter with $\mathbf{a}_{k}^{T} \mathbf{e}_{k}(z)$ and $-\tilde{\mathbf{a}}_{k}^{T} \mathbf{e}_{k}(z)$ as the first and second polyphase components, i.e., $H_{\left[\mathbf{a}_{k}: a\right]}(z)=\mathbf{a}_{k}^{T} \mathbf{e}_{k}\left(z^{2}\right)-z^{-1} \tilde{\mathbf{a}}_{k}^{T} \mathbf{e}_{k}\left(z^{2}\right) \cdot \mathcal{S}_{\left[\mathbf{a}_{k}: l\right]}$ denotes a $(k+l+1) \times(l+1)$ matrix derived from the $(k+1) \times 1$ vector $\mathbf{a}_{k}$ given by

$$
\left\{\mathcal{S}_{\left[\mathbf{a}_{k}: l\right]}\right\}_{i, j}= \begin{cases}a_{i-j}, & \text { when } 0 \leq i-j \leq k \\ 0, & \text { otherwise. }\end{cases}
$$

The matrix $\mathcal{S}_{\left[\mathbf{a}_{k}: l\right]}$ is commonly known as the convolution matrix. Consider the polynomial $C_{l}(z)=\sum_{i=0}^{l} z^{-i} c_{i}=$ $\mathbf{c}_{l}^{T} \mathbf{e}_{l}(z)$. Then the product $\mathbf{a}_{k}^{T} \mathbf{e}_{k}(z) \tilde{\mathbf{c}}_{l}^{T} \mathbf{e}_{l}(z)$ becomes $\left(\mathcal{S}_{\left[\mathbf{a}_{k}: l\right]} \tilde{\mathbf{c}}_{l}\right)^{T} \mathbf{e}_{k+l+1}(z)$.

\section{The SEQUENTIAL CharaCterizATION}

Let $H_{0}(z)$ and $H_{1}(z)$ be the symmetric and antisymmetric filters of lengths $2 k+2$ and $2 l+2$, respectively. They should be of the form, $H_{0}(z)=H_{\left[\mathbf{p}_{k}: s\right]}(z)$ and $H_{1}(z)=H_{\left[\mathbf{q}_{l}: a\right]}(z)$, for some $(k+1) \times 1$ and $(l+1) \times 1$ vectors $\mathbf{p}_{k}$ and $\mathbf{q}_{l}$, respectively. The corresponding polyphase matrix is given by

$$
\mathbf{E}_{\mathbf{p}_{k}, \mathbf{q}_{l}}(z)=\left[\begin{array}{rr}
\mathbf{p}_{k}^{T} \mathbf{e}_{k}(z) & \tilde{\mathbf{p}}_{k}^{T} \mathbf{e}_{k}(z) \\
\mathbf{q}_{l}^{T} \mathbf{e}_{l}(z) & -\tilde{\mathbf{q}}_{l}^{T} \mathbf{e}_{l}(z)
\end{array}\right]
$$

We will call the vectors $\mathbf{p}_{k}$ and $\mathbf{q}_{l}$ also as the polyphase components. From the form of the polyphase matrix, we can verify the following:

$$
\mathbf{E}_{\mathbf{p}_{k}, \mathbf{q}_{l}}(z)=\left[\begin{array}{rr}
1 & 0 \\
0 & -1
\end{array}\right]\left[\begin{array}{cc}
z^{-k} & 0 \\
0 & z^{-l}
\end{array}\right] \mathbf{E}_{\mathbf{p}_{k}, \mathbf{q}_{l}}\left(z^{-1}\right)\left[\begin{array}{cc}
0 & 1 \\
1 & 0
\end{array}\right] \text {. }
$$

This matrix forms a type $A$ system if the determinant is a monomial. Let $c z^{-p_{d}}$ be the determinant of the polyphase matrix $\mathbf{E}_{\mathbf{p}_{k}, \mathbf{q}_{l}}(z)$. From (2) we get

$$
p_{d}=(k+l) / 2 \text {. }
$$

This verifies that $k+l$ should be even. In other words, the difference in length should be a multiple of four. Equating the determinant in (1) and ignoring the scale factor, we get

$$
\mathbf{p}_{k}^{T} \mathbf{e}_{k}(z) \tilde{\mathbf{q}}_{l}^{T} \mathbf{e}_{l}(z)+\tilde{\mathbf{p}}_{k}^{T} \mathbf{e}_{k}(z) \mathbf{q}_{l}^{T} \mathbf{e}_{l}(z)=z^{-(k+l) / 2} .
$$

It can be verified that when $H_{0}(z)$ is antisymmetric and $H_{1}(z)$ is symmetric, i.e., when $H_{0}(z)=H_{\left[\mathbf{p}_{k}: a\right]}(z)$ and $H_{1}(z)=$ $H_{\left[\mathbf{q}_{l}: s\right]}(z)$, the condition for the filter pair to form a type $A$ system is given by the same equation.

In the remainder of this section, we will develop a sequential characterization of type $A$ filter banks. In Section II-A, given a symmetric or antisymmetric even length linear-phase filter, we find the expression for the complementary even-length linearphase filter (which should be of opposite symmetry) forming a type $A$ system if it exists. In Section II-B, we discuss about the condition on the first filter for the existence of a complementary linear-phase filter.

\section{A. PR Condition for Complementary Filter}

Let $\mathbf{p}_{k}$ be the polyphase component of the first filter and $\mathbf{q}_{l}$ be the polyphase component of the complementary filter. Equation (3) can be equivalently written as

$$
\begin{aligned}
& \left(\mathcal{S}_{\left[\mathbf{p}_{k}: l\right]} \tilde{\mathbf{q}}_{l}\right)^{T} \mathbf{e}_{k+l+1}(z)+\left(\mathcal{S}_{\left[\tilde{\mathbf{p}}_{k}: l\right]} \mathbf{q}_{l}\right)^{T} \mathbf{e}_{k+l+1}(z) \\
& \quad=\mathbf{b}_{k, l}^{T} \mathbf{e}_{k+l+1}(z)
\end{aligned}
$$

where $\mathbf{b}_{k, l}$ is the $(k+l+1) \times 1$ vector with the central element equal to unity and all other elements equal to zero. Now, the following can be verified:

$$
\mathcal{S}_{\left[\tilde{\mathbf{p}}_{k}: l\right]}=\mathbf{J}_{k+l+1} \mathcal{S}_{\left[\mathbf{p}_{k}: l\right]} \mathbf{J}_{l+1} \quad \tilde{\mathbf{q}}_{l}=\mathbf{J}_{l+1} \mathbf{q}_{l} .
$$

Substituting the above relations in (4), we get

$$
\mathcal{S}_{\left[\mathbf{p}_{k}: l\right]} \tilde{\mathbf{q}}_{l}+\mathbf{J}_{k+l+1} \mathcal{S}_{\left[\mathbf{p}_{k}: l\right]} \tilde{\mathbf{q}}_{l}=\mathbf{b}_{k, l} .
$$

From the form of $\mathrm{b}_{k, l}$, we can observe that the above equation is satisfied if and only if the vector $\mathcal{S}_{\left[\mathbf{p}_{k}: l\right]} \tilde{\mathbf{q}}_{l}$ is antisymmetric, except the central element is 0.5 . Equivalently, the sum of $i$ th and $(k+l-i)$ th elements in the vector $\mathcal{S}_{\left[\mathbf{p}_{k}: l\right]} \tilde{\mathbf{q}}_{l}$ should be zero for $i=0, \ldots,(k+l) / 2-1$, or the vector $\left[\mathbf{I}_{(k+l) / 2} \mathbf{0}_{(k+l) / 2,1} \mathbf{J}_{(k+l) / 2}\right] \mathcal{S}_{\left[\mathbf{p}_{k}: l\right]} \tilde{\mathbf{q}}_{l}$ should be zero. Additionally, the element at the center, given by $\mathbf{b}_{k, l}^{T} \mathcal{S}_{\left[\mathbf{p}_{k}: l\right]} \tilde{\mathbf{q}}_{l}$, should be 0.5 . Let $\hat{\mathbf{b}}_{k, l}=\left[\begin{array}{llll}0 & 0 & \ldots & 1\end{array}\right]^{T}$. The above condition can then be restated as

$$
\left[\begin{array}{c}
{\left[\mathbf{I}_{(k+l) / 2} \mathbf{0}_{(k+l) / 2,1} \mathbf{J}_{(k+l) / 2}\right]} \\
\mathbf{b}_{k, l}^{T}
\end{array}\right] \mathcal{S}_{\left[\mathbf{p}_{k}: l\right]} \tilde{\mathbf{q}}_{l}=\hat{\mathbf{b}}_{k, l} .
$$

For a $(k+1) \times 1$ vector, $\mathbf{d}_{k}$, define

$$
\mathcal{G}_{\left[\mathbf{d}_{k}: l\right]}=\left[\begin{array}{c}
{\left[\mathbf{I}_{(k+l) / 2} \mathbf{0}_{(k+l) / 2,1} \mathbf{J}_{(k+l) / 2}\right]} \\
\mathbf{b}_{k, l}^{T}
\end{array}\right] \mathcal{S}_{\left[\mathbf{d}_{k}: l\right]} .
$$

The above condition then becomes

$$
\mathcal{G}_{\left[\mathbf{p}_{k}: l\right]} \tilde{\mathbf{q}}_{l}=\hat{\mathrm{b}}_{k, l} .
$$

In other words $\tilde{\mathbf{q}}_{l} \in \mathcal{X}_{\left[\mathbf{p}_{k}: l\right]}$ where $\mathcal{X}_{\left[\mathbf{p}_{k}: l\right]}$ is the solution space for $\mathrm{x}$ in the equation

$$
\mathcal{G}_{\left[\mathbf{p}_{k}: l\right]} \mathbf{x}=\hat{\mathbf{b}}_{k, l} .
$$

The above discussion is summarized in the following.

$P R$ Condition for Complementary Filter: Let $\mathbf{p}_{k}$ and $\mathbf{q}_{l}$ be some $(k+1) \times 1$ and $(l+1) \times 1$ vectors, respectively, such that the 
filters $H_{\left[\mathbf{q}_{\ell}: a\right]}(z)$ and $H_{\left[\mathbf{q}_{\ell}: s\right]}(z)$ become complementary filters to the filters $H_{\left[\mathbf{p}_{k}: s\right]}(z)$ and $H_{\left[\mathbf{p}_{k}: a\right]}(z)$, respectively, forming type $A$ systems. $\tilde{\mathbf{q}}_{l}$ then lies in the solution space for $\mathbf{x}$ in (6), $\mathcal{X}_{\left[\mathbf{p}_{k}: l\right]}$, where $\mathcal{G}_{\left[\mathbf{p}_{k}: l\right]}$ is as defined in (5). Conversely, let $\mathbf{p}_{k}$ and $\mathbf{q}_{l}$ be some $(k+1) \times 1$ and $(l+1) \times 1$ vectors, respectively, and let $\tilde{\mathbf{q}}_{l}$ lie in the solution space for $\mathbf{x}$ in (6), $\mathcal{X}_{\left[\mathbf{p}_{k}: l\right]}$. The filters $H_{\left[\mathbf{q}_{l}: a\right]}(z)$ and $H_{\left[\mathbf{q}_{l}: s\right]}(z)$ then become complementary filters to the filters $H_{\left[\mathbf{p}_{k}: s\right]}(z)$ and $H_{\left[\mathbf{p}_{k}: a\right]}(z)$, respectively, forming type $A$ systems.

\section{B. Condition on the First Filter}

We will now consider the condition on $\mathbf{p}_{k}$ for the existence of a solution for (6). It is known that [5] if the polyphase component of the first filter (either symmetric or antisymmetric), $P_{k}(z)=\sum_{i=0}^{k} z^{-i} p_{i}$ does not have roots on the unit circle, there exists an unique polynomial $R_{k}(z)=\sum_{i=0}^{k} z^{-i} r_{i}$ of the same length such that it forms the polyphase component of the complementary filter (of opposite symmetry) forming a type $A$ filter bank. Let the first filter be symmetric and the complementary filter be antisymmetric. The corresponding polyphase matrix is given by

$$
\mathbf{E}_{P_{k}, R_{k}}(z)=\left[\begin{array}{rr}
P_{k}(z) & \tilde{P}_{k}(z) \\
R_{k}(z) & -\tilde{R}_{k}(z)
\end{array}\right] .
$$

The following matrix again forms a type $A$ system:

$$
\begin{aligned}
\mathbf{E}_{P_{k}, l}(z) & =\left[\begin{array}{cc}
1 & 0 \\
\Gamma_{l-k}(z) & z^{-(l-k) / 2}
\end{array}\right]\left[\begin{array}{cc}
P_{k}(z) & \tilde{P}_{k}(z) \\
R_{k}(z) & -\tilde{R}_{k}(z)
\end{array}\right] \\
& =\left[\begin{array}{cc}
P_{k}(z) & \tilde{P}_{k}(z) \\
Q_{l}(z) & S_{l}(z)
\end{array}\right]
\end{aligned}
$$

where $l-k$ is any positive even integer and $\Gamma_{l-k}(z)$ is a length $l-k+1$ antisymmetric polynomial. To verify that, it is first observed that the determinant of the above matrix is a monomial. Hence, it only remains to verify that $S_{l}(z)=-z^{-l} Q_{l}\left(z^{-1}\right)$. Since

$$
\begin{aligned}
Q_{l}(z) & =\Gamma_{l-k}(z) P_{k}(z)+z^{-(l-k) / 2} R_{k}(z) \\
S_{l}(z) & =\Gamma_{l-k}(z) \tilde{P}_{k}(z)-z^{-(l-k) / 2} \tilde{R}_{k}(z)
\end{aligned}
$$

it follows that

$$
\begin{aligned}
-z^{-l} Q_{l}\left(z^{-1}\right)= & \left(z^{-(l-k)} \Gamma_{l-k}\left(z^{-1}\right)\right)\left(z^{-k} P_{k}\left(z^{-1}\right)\right) \\
& -z^{-l}\left(z^{(l-k) / 2} R_{k}\left(z^{-1}\right)\right) \\
= & -\left(z^{-(l-k)} \Gamma_{l-k}\left(z^{-1}\right)\right)\left(\tilde{P}_{k}(z)\right) \\
& -z^{-(l-k) / 2} \tilde{R}_{k}(z) .
\end{aligned}
$$

Since $\Gamma_{l-k}(z)$ is antisymmetric, $-z^{-(l-k)} \Gamma_{l-k}\left(z^{-1}\right)=$ $\Gamma_{l-k}(z)$. Hence, $S_{l}(z)=-z^{-l} Q_{l}\left(z^{-1}\right)$. This verifies that if the polyphase component of the first symmetric filter does not have roots on the unit circle, an antisymmetric complementary filter of higher length forming a type $A$ system also exists. In a similar way, we can verify that if the first filter is antisymmetric with polyphase component satisfying the above condition, there exists a symmetric complementary filter of higher length forming a type $A$ system. Hence, for unequal lengths, restricting the complementary filter to be longer than the given filter does not lead to any loss of generality.
To sum up, if the polyphase component of the first filter $P_{k}(z)$ does not have roots on the unit circle, there always exists a polynomial of length $l+1$, where $l-k$ is a nonnegative even integer, such that it forms the polyphase component of a complementary filter forming a type $A$ system. In other words, if the polynomial $\mathbf{p}_{k}^{T} \mathbf{e}_{k}(z)$ does not have roots on the unit circle, for a proper $l$, the solution space $\mathcal{X}_{\left[\mathbf{p}_{k}: l\right]}$ of (6) is nonempty such that for a vector $\tilde{\mathbf{q}}_{l} \in \mathcal{X}_{\left[\mathbf{p}_{k}: l\right]}$, the filters $H_{\left[\mathbf{q}_{l}: a\right]}(z)$ and $H_{\left[\mathbf{q}_{l}: s\right]}(z)$ become complementary filters to the filters $H_{\left[\mathbf{p}_{k}: s\right]}(z)$ and $H_{\left[\mathbf{p}_{k}: a\right]}(z)$, respectively, forming type $A$ systems.

\section{The Proposed Design Method}

Using the perfect reconstruction condition in the form of a system of linear equations derived in the previous section, a sequential method is adopted for the design of type $A$ filter banks. The idea is to first choose an even-length filter such that there exists a complementary filter of equal or higher even length forming a type $A$ system. The second filter is chosen using the remaining degrees of freedom if any. The procedure is iterated leading to a joint optimization of both the filters.

In filter bank design via optimization, it is customary to construct a cost function, which takes the set of parameters involved in the characterization as input. From the parameter values, the filters are computed. From the filters, it computes and returns the performance measure. A nonlinear optimization algorithm is used to optimize the cost function leading to the desired filter bank. Given below is the algorithm for the cost function used in our sequential approach, which we refer to as the convolution matrix method for obvious reasons. The cost function takes the parameter vector $\hat{\mathbf{v}}_{k}$ corresponding to the polyphase component of the first filter to be designed. $\hat{\mathbf{v}}_{k}$ is initialized to the vector corresponding to the polyphase component of a linear phase length $2 k+2$ initialization filter of some desired response $H_{v}(z)$. Additionally, when $k<l$, the function takes another vector $\mathbf{g}_{l}$ as the input, which is the vector corresponding to the polyphase component of another linear phase filter of length $2 l+2$ having some desired response $H_{u}(z)$ (if $H_{v}(z)$ is symmetric $H_{u}(z)$ should be antisymmetric and vice versa). In Step $1, \mathbf{v}_{k}$ is the vector obtained by applying a perturbation on $\hat{\mathbf{v}}_{k}$ such that $\mathbf{v}_{k}^{T} \mathbf{e}_{k}(z)$ has no root on the unit circle.

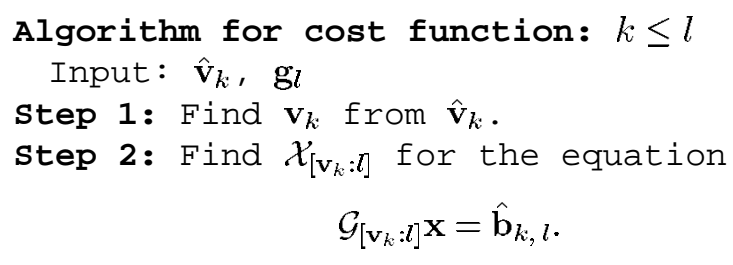

If $k=l$, find the unique vector $\hat{\mathbf{x}} \in \mathcal{X}_{\left[v_{k}: l\right]}$. Else if $k<l$, find $\hat{\mathbf{x}}$, the least square approximation of $\tilde{\mathrm{g}}_{l}$ in $\mathcal{X}_{\left[\mathrm{v}_{k}: l\right]}$. Set $\mathbf{u}_{l}=$ $\mathbf{J}_{l+1} \hat{\mathbf{x}}$.

Step 3: If $H_{v}(z)$ is symmetric, find the type $A$ system $\mathbf{h}(z)=\left[\begin{array}{ll}H_{\left[\mathrm{v}_{k}: s\right]}(z) & H_{\left[\mathbf{u}_{l}: a\right]}(z)\end{array}\right]^{T}$. Else if $H_{v}(z)$ is antisymmetric, find the type $A$ system $\mathbf{h}(z)=\left[H_{\left[\mathbf{v}_{k}: a\right]}(z) H_{\left[\mathbf{u}_{l}: s\right]}(z)\right]^{T}$.

Step 4: Find the cost $\mathcal{C}(\mathbf{h}(z))$, which is the performance measure of $\mathbf{h}(z)$. 

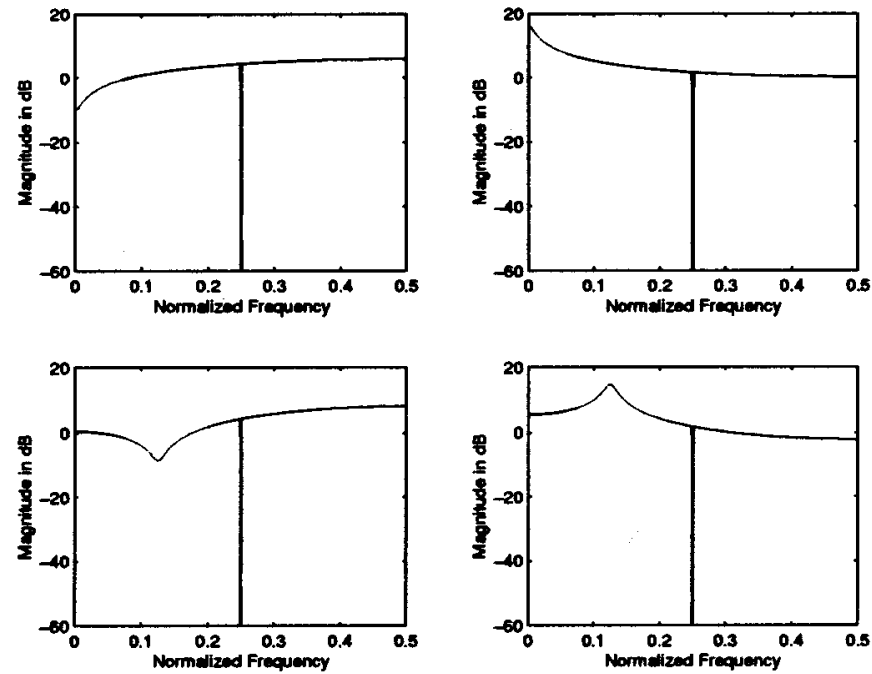

Fig. 1. Responses of the ideal filter banks. Top left: Analysis response for $\mathrm{AR}$ (1) source; top right: Synthesis response for AR(1) source; bottom left: Analysis response for AR(2) source; bottom right: Synthesis response for $\operatorname{AR}(2)$ source.

Note that in the case of unequal lengths, the degrees of freedom in choosing the longer filter with the shorter filter chosen are not considered in the iteration. If considered, the effect of the corresponding parameters on the cost function heavily depends upon the parameters corresponding to the first (shorter) filter. This leads to convergence problem in optimization.

\section{DESIGN EXAMPLES}

We used quasi-Newton algorithm for optimizing the cost function. Various performance measures (Step 4 of the algorithm) were used in the cost function.

\section{A. Optimization for Subband Coding}

In the optimization for subband coding, the performance measure used is the mean square deviation of the filer bank response from that of the ideal filter bank (in the sense of maximum subband coding gain) [12] for a given source.

The sources considered are as follows:

- $\operatorname{AR}(1)$ source with $\rho=0.95$.

- AR(2) source with $r=0.95$ and $\theta=\pi / 4$.

Frequency responses of the ideal filter banks for these sources are given in Fig. 1.

Example 1-AR(1) Source: Coding gain of the ideal filter bank for this source is 6.56. Table I gives the coding gains of the designed filter banks with equal length filters of lengths 10 , 20,30 , and 40 optimized for AR(1) source using the convolution matrix method. Coding gains of the filter banks obtained using the lattice method [1] are given for comparison. The total number of flops taken for the optimization in each case is also tabulated. Fig. 2 gives the analysis and synthesis responses of the length 20 filter banks obtained using both the convolution matrix and the lattice methods. In the case of filter banks obtained using the lattice method, it is observed that both the stopband attenuation and the passband shaping are less than that of
TABLE I

CODING GAINS FOR THE FILTER BANKS OPTIMIZED FOR AR(1) SOURCE

\begin{tabular}{c|l|l|l}
\hline Method & Length & Gain & Flops \\
\hline Convolution & 10 & 4.83 & $4.0316 \times 10^{8}$ \\
\cline { 2 - 4 } Matrix & 20 & 5.09 & $2.4664 \times 10^{9}$ \\
\cline { 2 - 4 } Method & 30 & 5.32 & $1.4555 \times 10^{10}$ \\
\cline { 2 - 4 } & 40 & 5.41 & $1.6958 \times 10^{10}$ \\
\hline Lattice & 10 & 4.13 & $1.8576 \times 10^{8}$ \\
\cline { 2 - 4 } Method & 20 & 4.18 & $9.6531 \times 10^{8}$ \\
\cline { 2 - 4 } & 30 & 4.03 & $2.2865 \times 10^{9}$ \\
\cline { 2 - 4 } & 40 & 4.00 & $5.5280 \times 10^{9}$ \\
\hline
\end{tabular}
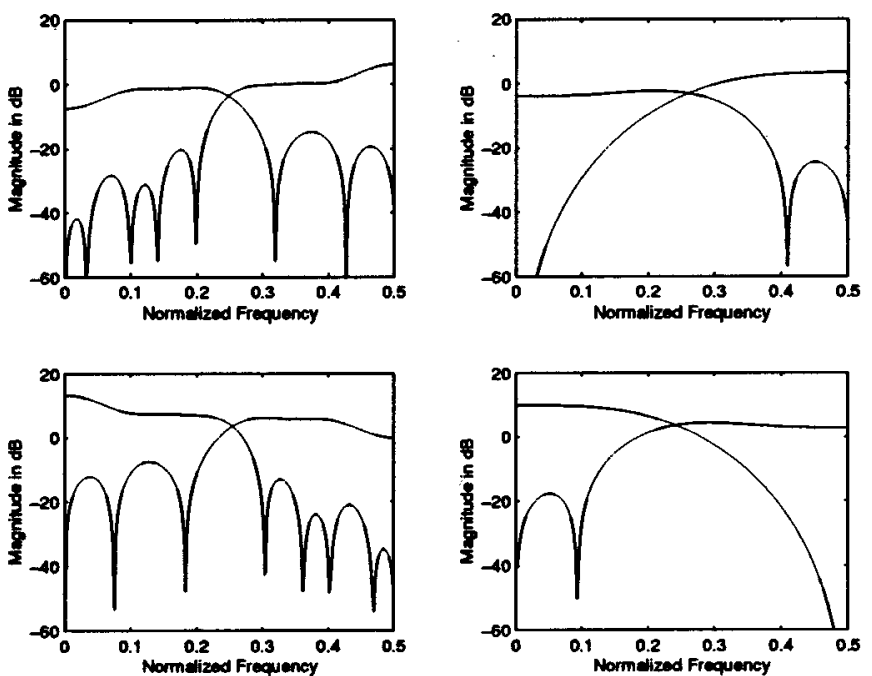

Fig. 2. Responses of the filter banks with equal length filters of length 20 optimized for AR(1) source. Top left: Analysis filter bank obtained using convolution matrix method; bottom left: Corresponding synthesis filter bank; top right: Analysis filter bank obtained using lattice method; bottom right: Corresponding synthesis filter bank.

the filter banks of the same length obtained by the proposed convolution matrix method. Hence, the coding gains are also less than that of the filter banks obtained by the convolution matrix method. This is due to the fact that the parameters in convolution matrix method are the filter coefficients themselves and, hence, the overall cost function is less complex compared to the lattice method. It is also observed that in the case of filter banks obtained by the convolution matrix method, there is no significant change in the shaping of the passband with the increase in the length. However, the stopband attenuation increases with the length. Due to the increase in the stopband attenuation, coding gain also increases with the length, but the improvement is not significant.

Table II gives coding gains of the filter banks optimized for the same source with unequal length filters with the average length of 20 . The length pairs considered are $(8,32),(16,24)$, $(24,16)$, and $(32,8)$. The first entry represents the length of the symmetric filter and the second entry represents the length of the antisymmetric filter. Note that the symmetric filter is used for low pass and the antisymmetric filter is used for high pass. Comparing with the equal length filter bank of length 20 in the Table I, first three cases in Table II give similar performance, but the coding gain of the fourth is slightly lower. A possible reason is that since most of the energy in the AR(1) source is 
TABLE II

Coding Gains of UneQual LENGTH FILTER BANKS OPTIMIZED For AR(1) SOURCE Using THE CONVOLUTION MATRIX METHOD

\begin{tabular}{c|l|l}
\hline Lengths & Gain & Flops \\
\hline 8,32 & 4.96 & $1.8420 \times 10^{8}$ \\
\hline 16,24 & 5.11 & $1.4694 \times 10^{9}$ \\
\hline 24,16 & 5.16 & $1.4835 \times 10^{9}$ \\
\hline 32,8 & 4.35 & $5.0970 \times 10^{8}$ \\
\hline
\end{tabular}

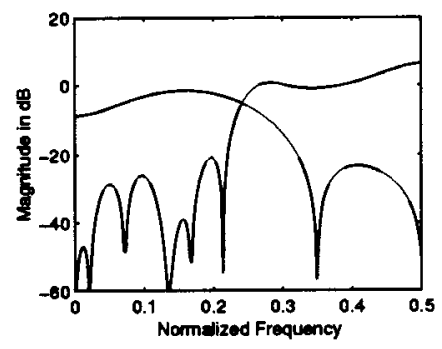

Fig. 3. Response of the analysis filter bank with filter lengths 8 and 32 optimized for AR(1) source using the convolution matrix method.

TABLE III

CODING GAINS FOR THE FILTER BANKS OPTIMIZED FOR AR(2) SOURCE

\begin{tabular}{c|l|l|l}
\hline Method & Length & Gain & Flops \\
\hline \multirow{3}{*}{$\begin{array}{c}\text { Convolution } \\
\text { Matrix }\end{array}$} & 10 & 5.60 & $3.8500 \times 10^{9}$ \\
\cline { 2 - 4 } Method & 20 & 6.09 & $2.0933 \times 10^{10}$ \\
\cline { 2 - 4 } & 30 & 6.41 & $7.7716 \times 10^{10}$ \\
\cline { 2 - 4 } & 40 & 6.63 & $1.9013 \times 10^{11}$ \\
\hline \multirow{3}{*}{$\begin{array}{l}\text { Lattice } \\
\text { Method }\end{array}$} & 10 & 4.67 & $0.9317 \times 10^{8}$ \\
\cline { 2 - 4 } & 20 & 4.72 & $2.9283 \times 10^{9}$ \\
\cline { 2 - 4 } & 30 & 5.11 & $2.2409 \times 10^{8}$ \\
\cline { 2 - 4 } & 40 & 5.28 & $3.2523 \times 10^{9}$ \\
\hline
\end{tabular}

concentrated in the low-pass side, reduction in the stopband attenuation in the high-pass filter leads to higher geometric mean of the subband variances. Fig. 3 gives the analysis response corresponding to the length pair $(8,32)$.

Example 2-AR(2) Source: Table III compares the coding gains of the filter banks optimized for AR(2) source obtained using the convolution matrix method to that of the lattice method. The number of flops in each case is also tabulated. The coding gain for the ideal filter bank is 7.65. In this case, as seen in the table, filter banks also obtained by the convolution matrix method perform better than that of lattice method due to the same reason mentioned in the previous example. In the case of filter banks obtained using the convolution matrix method, it is observed that both the passband shaping and the stopband attenuation improve with the increase in the length. This is due to the fact that the required shaping for this case is more complex compared to the previous example. Due to this factor, improvement in the coding gain with the increase in length is more significant compared to the previous example. Fig. 4 gives responses of length 20 analysis filter banks obtained using both the convolution matrix and the lattice methods.

Table IV gives the coding gains of unequal length filter banks optimized for the same source. The length combinations considered are the same as in the previous example. The entries in
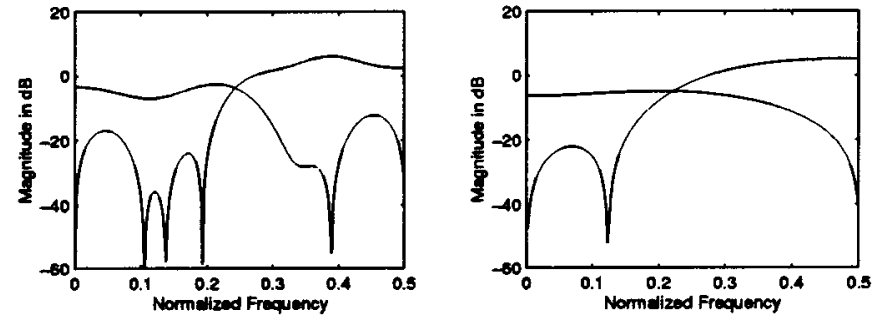

Fig. 4. Responses of the analysis filter banks with equal length filters of length 20 optimized for AR(2) source. Left: Filter bank obtained by convolution matrix method; right: Filter bank obtained by lattice method.

TABLE IV

CODing Gains of Unequal Length Filter BanKs Optimized For AR(2) SOURCE Using THE CONVOLUTION MATRIX METHOD

\begin{tabular}{c|l|l}
\hline Lengths & Gain & Flops \\
\hline 8,32 & 5.58 & $1.8367 \times 10^{8}$ \\
\hline 16,24 & 5.98 & $1.3746 \times 10^{9}$ \\
\hline 24,16 & 5.04 & $8.6550 \times 10^{8}$ \\
\hline 32,8 & 5.98 & $5.6308 \times 10^{8}$ \\
\hline
\end{tabular}

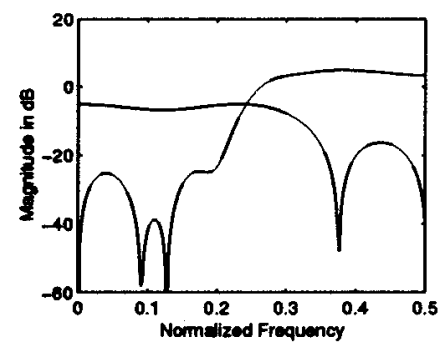

Fig. 5. Responses of the analysis filter bank with filter lengths 8 and 32 optimized for $\mathrm{AR}(2)$ source using the convolution matrix method.
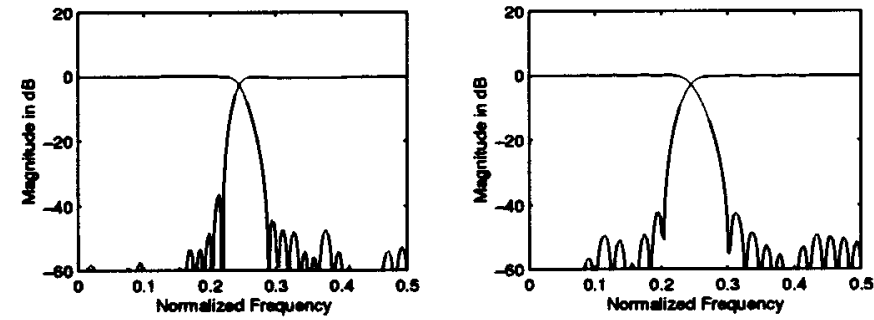

Fig. 6. Responses of equal length filter banks of length 64 optimized for stopband energy. Left: Filter bank obtained using convolution matrix method; Right: Filter bank obtained using lattice method.

the length pairs have the same interpretation as in the first example. Fig. 5 gives the response of the analysis filter bank corresponding to length pair $(8,32)$ obtained by the convolution matrix method.

\section{B. Optimization for Conventional Response}

Example 3-Stopband Energy: The performance used here is given by $E_{s b e}=\int_{\pi / 2}^{\pi}\left|H_{0}\left(e^{j \omega}\right)\right|^{2} d \omega+\int_{0}^{\pi / 2}\left|H_{1}\left(e^{j \omega}\right)\right|^{2} d \omega$. Fig. 6 compares the stopband energy of equal-length filter bank of length 64 obtained by the convolution matrix method with that of the filter bank of same length and same type obtained by the lattice method. It is clear from the figure that the filter bank 
TABLE V

Performance Comparison of Length 22 Egual Length Filter Banks

\begin{tabular}{c|c|c|c|c|c}
\hline \hline Method & $E_{p b d 0}$ & $\begin{array}{c}E_{\text {sbe0 }} \\
\times 10^{-5}\end{array}$ & $\begin{array}{c}E_{p b d 1} \\
\times 10^{-5}\end{array}$ & $\begin{array}{c}E_{\text {sbe } 1} \\
\times 10^{-3}\end{array}$ & $\begin{array}{c}\Phi \\
\times 10^{-1}\end{array}$ \\
\hline Convol. & 1.0149 & 2.8733 & 7.7980 & 0.2806 & 1.6163 \\
\hline LN & 1.0149 & 1.9296 & 5.7431 & 1.0719 & 1.6182 \\
\hline \hline
\end{tabular}

obtained by the convolution matrix method has lower stopband energy.

Example 4-Stopband Energy and Passband Deviation: A length 22 equal length filter bank is designed using the following performance measure:

$$
\begin{aligned}
\mathbf{\Phi}= & \alpha_{0} / 2 \pi\left[E_{p b d 0}+\alpha_{s 0} E_{s b e 0}\right] \\
& +\alpha_{1} / 2 \pi\left[E_{p b d 1}+\alpha_{s 1} E_{s b e 1}\right]
\end{aligned}
$$

where

$$
\begin{aligned}
& E_{p b d 0}=\int_{0}^{\omega_{p 0}}\left|1-H_{0}\left(e^{j \omega}\right)\right|^{2} d \omega \\
& E_{s b e 0}=\int_{\omega_{s 0}}^{\pi}\left|H_{0}\left(e^{j \omega}\right)\right|^{2} d \omega \\
& E_{p b d 1}=\int_{\omega_{p 1}}^{\pi}\left|1-H_{1}\left(e^{j \omega}\right)\right|^{2} d \omega
\end{aligned}
$$

and

$$
E_{s b e 1}=\int_{0}^{\omega_{s 1}}\left|H_{1}\left(e^{j \omega}\right)\right|^{2} d \omega
$$

the integral limits denote usual band edges. In order to compare with the Lagrange-Newton (LN) method design example presented in [10] (this example is shown to outperform [1]), we used identical values $\omega_{p 0}=\omega_{s 1}=0.4 \pi, \omega_{p 1}=\omega_{s 0}=0.6 \pi$, $\alpha_{0}=1, \alpha_{1}=2, \alpha_{s 0}=1$, and $\alpha_{s 1}=0.8$. With the above settings, performance of the filter bank designed with the convolution matrix method is compared with that of the design example of [10] in the Table V. There is marginal improvement in the total cost using the proposed method. However, as mentioned previously, in the LN method, the constraint equations have to be derived for each combination of filter lengths, whereas in the convolution matrix method, the system of equations has a general form with respect to any valid combinations of filter lengths.

\section{CONCLUSION}

In this paper, a sequential approach for the design of type $A$ two-channel linear phase perfect reconstruction filter banks has been discussed. The perfect reconstruction problem, which involves polynomials, was formulated in terms of the convolution matrix. The perfect reconstruction condition was then obtained in the form of a system of linear equations. Using the condition, an algorithm for the design in a sequential fashion has been presented. The method allows design of filter banks by searching the entire class. It also allows control over the individual lengths of the filters. The effectiveness of the approach over lattice and $\mathrm{LN}$ methods is discussed and demonstrated with design examples.

\section{REFERENCES}

[1] T. Q. Nguyen and P. P. Vaidyanathan, "Two-channel perfect reconstruction FIR QMF structures which yield linear phase FIR analysis and synthesis filters," IEEE Trans. Acoust., Speech, Signal Processing, vol. 37, pp. 676-690, May 1989.

[2] M. Vetterli and D. Le Gall, "Perfect reconstruction FIR filter banks: Some properties and factorizations," IEEE Trans. Acoust., Speech Signal Processing, vol. 37, pp. 1057-1071, July 1989.

[3] C. K. Goh and Y. C. Lim, "Novel approach for the design of two-channel perfect reconstruction linear phase FIR filter banks," IEEE Trans. Circuits Syst. II, vol. 45, pp. 1141-1146, Aug. 1998.

[4] P. Saghizadeh and A. N. Willson, Jr., "Using unconstrained optimization in the design of two-channel perfect reconstruction linear phase FIR filter banks," in Proc. IEEE Midwest Symp. Circuits Syst., vol. 2, Aug. 1994, pp. 1053-1056.

[5] E. Kofidis, S. Theodoridis, and N. Kalouptsidis, "On the perfect reconstruction problem in N-band multirate maximally decimated FIR filter banks," IEEE Trans. Signal Processing, vol. 44, pp. 2413-2423, Oct. 1996.

[6] H. Xu, W.-S. Lu, and A. Antoniou, "Design of perfect reconstruction QMF banks by a null space projection method," in Proc. IEEE ICASSP, vol. 2, 1995, pp. 965-968.

[7] W.-S. Lu, "Design of perfect reconstruction QMF banks: A parameterization method," in Proc. Asilomar Conf. Signals, Syst. Comput., vol. 2, 1996, pp. 1329-1333.

[8] D. Pinchon and P. Siohan, "Analysis, design and implementation of twochannel linear phase filter banks: A new approach," IEEE Trans. Signal Processing, vol. 46, pp. 1814-1826, July 1998.

[9] B. R. Horng and A. N. Willson Jr., "The design of multiplierless twochannel linear phase FIR filter banks with application to image subband coding," in Proc. IEEE ISCAS, vol. 1, 1990, pp. 650-653.

[10] — - "Lagrange multiplier approaches to the design of two-channel perfect-reconstruction linear phase FIR filter banks," IEEE Trans. Signal Processing, vol. 40, pp. 364-374, Feb. 1992.

[11] C. K. Chen, "A novel design technique for two-channel linear-phase perfect-reconstruction FIR filter banks," Signal Process., vol. 58, no. 3, pp. 261-271, May 1997.

[12] P. P. Vaidyanathan and A. Kirac, "Result on optimal biorthogonal filters banks," IEEE Trans. Circuits Systems II, vol. 45, pp. 932-947, Aug. 1998.

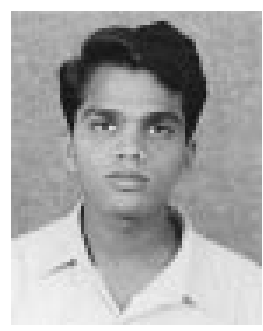

A. Muthuvel received the B.Tech. degree in electronics and communication engineering from Pondicherry Engineering College, Pondicherry, India, in 1995, and the M.Sc. degree in electrical communication engineering from the Indian Institute of Science, Bangalore, India, in 1999.

$\mathrm{He}$ is currently pursuing doctorate research at the Swiss Federal Institute of Technology, Lausanne, Switzerland. His main interest includes wavelets and its applications in biomedical image processing.

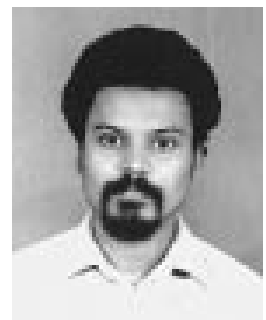

Anamitra Makur (SM'00) received the B.Tech. degree in electronics and electrical communication engineering from the Indian Institute of Technology, Kharagpur, in 1985 and the M.S. and Ph.D. degrees in electrical engineering from the California Institute of Technology, Pasadena, in 1986 and 1990.

$\mathrm{He}$ is currently an Associate Professor of Electrical Communication Engineering, Indian Institute of Science, Bangalore. His research interests in signal compression include subband coding and filterbank design, vector quantization, motion field coding, other image/video compression schemes and standards, and multimedia applications. His interests in image/video processing includes halftoning, image restoration, and 2-D filter design.

Dr. Makur is the recipient of the 1998 Young Engineer Award from the Indian National Academy of Engineering. 